\title{
Ion Enhancement Effect of Laser-Enhanced Ionization Induced by One-Step Excitation and Two-Step Excitation
}

\author{
KING-DO SU and KING-CHUEN LIN* \\ Department of Chemistry, National Taiwan University, Taipei, Taiwan 10764, and Institute of Atomic and Molecular Sciences, \\ Academia Sinica, P.O. Box 23-166, Taipei, Taiwan 10764, Republic of China
}

\begin{abstract}
The two-step laser-enhanced ionization (LEI) technique is believed to be more sensitive and selective in trace analysis than one-step LEI. As reported previously, for optimization of the two-step LEI configuration, factors involving laser intensity, transition probability of second-step excitation, collisional ionization rates, and transition linewidth should be taken into account. We have derived a simple theoretical model in relation to these parameters that is rough but suitable in practice for characterizing the ion yields enhanced by two-step LEI over one-step LEI. According to the model, each parameter affecting the ion enhancement has been systematically examined and compared to the theoretical evaluation. The findings of the relative ion enhancement as a function of each related parameter are consistent with the model prediction, except for the effect of transition linewidth, which is underestimated theoretically due to the difficulty of establishing the experimental conditions.
\end{abstract}

Index Headings: One-step laser-enchanced ionization; Two-step laserenhanced ionization; Trace analysis; Ion enhancement.

\section{INTRODUCTION}

The laser-enhanced ionization (LEI) technique has been thoroughly developed over the last decades to the point where it has become a "textbook" analytical tool, that is very powerful, especially in trace analysis. ${ }^{1}$ Recent exploration also suggests that this technique is as capable as other spectrometric methods in many fields. For instance, the LEI technique has been successfully employed to measure the ion lifetime, ${ }^{2}$ the free atoms released, ${ }^{3}$ and the atomization efficiency in a flame; ${ }^{4}$ to diagnose the ion mobility, diffusion coefficients, and temperature of a flame; ${ }^{5-7}$ and to determine the spectroscopic structures of atomic, molecular, and radical species, ${ }^{8-11}$ as well as to serve in the area of chromatography as a detector. ${ }^{12,13}$

The principle of LEI involves using a tunable dye laser to promote the population of an analyte to its excited state, and then monitoring the resultant ion yield.' According to the Boltzmann theory, the collisional ionization rate of the excited analyte is enhanced substantially over that in its ground state. For improving the sensitivity and selectivity of LEI utilized in trace analysis, efforts have been made to deal with issues related to the electrical interference, ${ }^{14-16}$ the electrode design, ${ }^{17,18}$ the ion collection configuration, ${ }^{19,20}$ and the influence of space charge and biased voltage on the ion collection efficiency. ${ }^{21,22}$ Among these concerns, employment of dual lasers through a stepwise excitation, causing more of the analyte to be promoted to its higher states, has proved to be a very efficient method to increase the ion yield. ${ }^{1,23-27}$ The col-

Received 31 March 1993; revision received 4 October 1993.

* Author to whom correspondence should be sent. lisional ionization rate is thus expected to increase exponentially with a decrease in the energy difference between the populated state and the ionization continuum. The question is whether the LEI process involving twostep excitation is superior to the approach using singlestep excitation. The answer seems to be positive. However, Havrilla and Choi found that in the LEI detection of $\mathrm{Zn}$ atoms in a flame via two-step excitation with a firststep ${ }^{1} S_{0} \rightarrow{ }^{1} P_{1}$ transition and second-step ${ }^{1} P_{1} \rightarrow{ }^{3} D_{1}$ (or ${ }^{3} D_{2}$ ) transition, the detection limit is five times as large as that with the first-step excitation alone. ${ }^{28}$ Obviously, changing the collisional ionization rate is not the only factor to affect the behavior of the ion yield. Since the LEI scheme with two-step excitation followed by collisional ionization has been popularly used in detecting trace analytes, it is of importance to characterize the ionization mechanisms and compare the ion yields produced between single-step excitation and two-step excitation.

Theoretical consideration of one-step and two-step LEI mechanisms based upon the rate equations has been thoroughly derived, with reliance upon several parameters related to the collisional ionization rates, quenching rates, transition probabilities, and laser spectral irradiance. ${ }^{27,29-32}$ Given these parameters, the ion yields produced between the two-step LEI and one-step LEI processes may be effectively compared. However, precisely measuring each individual parameter in a flame system is not trivial; a large error is possibly introduced because of uncertainty with respect to each parameter, and therefore theoretically comparing the ion yields induced by two-step LEI process with a one-step LEI process is somewhat difficult.

In a previous work, ${ }^{33}$ we used an apparatus analogous to that used for conventional LEI, but employed two laser beams in counter propagation to enhance the ionization rate. We have demonstrated the influence of second-step transition probability upon the sodium ion enhancement induced by two-step LEI over one-step LEI processes. ${ }^{33}$ As reported, ${ }^{29-32}$ to optimize the two-step LEI apparatus for increasing its sensitivity and selectivity, one must simultaneously take into account the factors related to the transition probability, the laser intensity, the collisional ionization rate, and the quenching effect of the transition states. In this paper, we extend our work to thoroughly examine each individual parameter that may cause enhancement of ion yield obtained by two-step over onestep LEI. The data are then compared to their theoretical counterpart-a simple model based upon the steady-state approximation that is rough but is suitable in practice for characterizing the ion yield enhanced by the two-step excitation process. In the following discussion, we briefly 
describe the experimental apparatus, then outline the theoretical modeling, and finally present our results and discussion.

\section{EXPERIMENTAL}

The experimental apparatus for one-step and two-step LEI experiments has been illustrated in our previous work. ${ }^{4,7,33}$ Only a brief account is given here.

For generating the atomic elements in a flame, we used a commercial burner head assembly (Perkin-Elmer) with a $100-\mathrm{mm} \times 0.5-\mathrm{mm}$ slot burner head, coupled with an interlocked gas control system by which acetylene $(0.5$ $\mathrm{L} / \mathrm{min}$ ) and air $(12.5 \mathrm{~L} / \mathrm{min})$ were premixed prior to reaching the burner head. The flame temperature measured approximately $2500 \mathrm{~K}$.?

Two tunable dye lasers (Quanta-Ray PDL-2) pumped simultaneously by an Nd:YAG laser (DCR-2A) were employed as the radiation sources, covering the wavelengths 420 to $795 \mathrm{~nm}$ with use of the appropriate laser dyes. The dye laser has a pulse duration of 5 to $8 \mathrm{~ns}$; its output power, varying in the range of 0.1 to $2 \mathrm{~mJ}$, was monitored by a surface absorbing disk calorimeter (Scientech 360001) throughout the experiment. The laser beam in the one-step LEI experiment was directed longitudinally through the flame at $7.5 \pm 0.5 \mathrm{~mm}$ above the burner head. In the two-step LEI experiment, the additional laser beam was directed from the opposite side with respect to the first laser beam. Two beams were overlapped temporally and spatially above the center of the burner head. The unfocused laser beams involved were collimated through an aperture $2.5 \pm 0.5 \mathrm{~mm}$ in diameter to allow for a homogeneous photon flow throughout the flame. In this manner, the scattering light was eliminated, so that the ionized portion of sample atoms probably caused by light scattering can be neglected. ${ }^{34}$

The resulting one-step and two-step LEI ions were collected with a pair of voltage-biased electrodes along the flame. The electrodes were set about $12 \pm 1 \mathrm{~mm}$ apart and suspended in the vicinity of the flame. The current obtained was amplified with a current-to-voltage converter (Keithley, Model 427) and then fed into a boxcar averager (PAR, Models 4402, 4420, and 4422) for signal processing. The result was displayed on an oscilloscope (Tektronix, Model 2445) or stored in a microcomputer for later data treatment.

$\mathrm{K}, \mathrm{Ca}$, and $\mathrm{Ba}$ salts were used as the reagents. Each aqueous salt solution was prepared for $100 \mathrm{ppm}(\mu \mathrm{g} / \mathrm{mL})$ and was then nebulized at a flow rate of $4.5 \mathrm{~mL} / \mathrm{min}$ into the flame. The linearity of concentration dependence of the LEI signal has been carefully checked in our experiments. ${ }^{35}$ The energy schemes of the two-step LEI process for $\mathrm{K}, \mathrm{Ca}$, and $\mathrm{Ba}$ atoms are shown in Fig. 1. The $\mathrm{K}$ atom was ionized through various stepwise processes which shared a common first-step excitation. In terms of this consideration, the transition probability related to the second-step excitation was examined for its role in the comparison of ionization enhancement between two-step LEI and one-step LEI. We also conducted the one-step and two-step LEI experiments using $\mathrm{Ca}$ as the reagent to determine the influence of laser intensity and collisional ionization rate. In the experiment to detect the dependence of ion enhancement upon transition linewidth, we
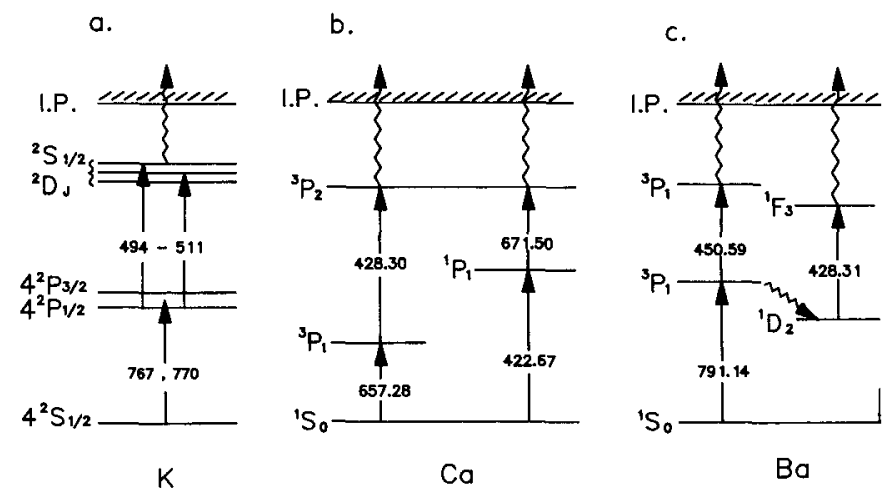

FIG. 1. Two-step LEI schemes for $\mathrm{K}, \mathrm{Ca}$, and $\mathrm{Ba}$ atoms.

compared the $\mathrm{Ba}$ ion yield induced between two different two-step LEI schemes. The first scheme is composed of stepwise excitation $6 s^{21} S_{0} \rightarrow 6 s 6 p^{3} P_{1}$ and $6 s 6 p^{3} P_{1} \rightarrow 6 p^{23} P_{1}$ transitions. In the second scheme the first-step excitation remained invariant, but the second-step was changed to the $6 s 5 d^{1} D_{2} \rightarrow 6 s 4 f^{1} F_{3}$ transition induced by the second laser, as optically delayed by 2 ns with respect to the first laser beam.

\section{THEORETICAL MODELING}

A four-level energetic system is considered in the twostep LEI scheme, where the first-step excitation is from the $N_{1}$ to $N_{2}$ state, the second-step excitation from the $N_{2}$ to $N_{3}$ state, and then the last step of collisional ionization from the $N_{3}$ to $N_{i}$ state. For derivation of the ion yield for two-step LEI, the following assumptions are adopted:

1. A steady-state approximation is applicable to the $N_{3}$ state; i.e., $\mathrm{d} N_{3} / \mathrm{d} t=0$.

2. The ion/electron recombination rate is slow and negligible during our laser pulse duration of 5 to $8 \mathrm{ns.}^{29}$

3. The laser profile is assumed to be as narrow as a delta function.

It is reasonable to use a steady-state approximation since the excitation rate to the $N_{3}$ state is small, as limited by the condition of undersaturation, while the collisional ionization rate from the excited state is relatively large, especially when $N_{3}$ is close to the ionization continuum. As soon as the intermediate state is populated, it can be depleted rapidly. This consideration suggests that a steadystate appoximation should be valid for the system. ${ }^{36,37}$ Under this appoximation, the population density of $N_{3}$ can be expressed as ${ }^{24,38}$

$$
\begin{aligned}
N_{3} & =k_{23} \tau_{3} N_{2} \\
& =\frac{\lambda_{23}{ }^{2} A_{32} I_{23}}{2 \Pi \Delta \omega_{23}} N_{2}
\end{aligned}
$$

where $\tau_{3}$ is the effective lifetime of the $N_{3}$ state; $I_{23}$, the laser intensity of the second-step excitation; $\lambda_{23}$, the excitation wavelength from the $N_{2}$ to $N_{3}$ state; $A_{32}$, the Einstein spontaneous emission coefficient; and $\Delta \omega_{23}$, the linewidth of the transition.

Because the ion/electron recombination rate is negligible, the ion yield $N_{i}$ (TLEI) induced by the two-step LEI is related to the population density of $N_{3}$ by

$$
N_{i}(\text { TLEI })=k_{3 i} N_{3} \Delta t
$$


where $k_{3 i}$ is the collisional ionization rate coefficient from $N_{3}$ to $N_{i}$, and $\Delta t$ is the laser pulse duration. Substitution of Eq. 1 yields

$$
N_{i}(\text { TLEI })=\frac{\lambda_{23}^{2} A_{32} I_{23} k_{3 i}}{2 \Pi \Delta \omega_{23}} N_{2} \Delta t
$$

Analogously, under the assumption of steady-state approximation and neglect of the ion/electron recombination, the ion yield $N_{i}$ (SLEI) for only single-step excitation may be expressed by

$$
N_{i}(\mathrm{SLEI})=\frac{\lambda_{12}^{2} A_{21} I_{12} k_{2 i}}{2 \Pi \Delta \omega_{12}} N_{1} \Delta t .
$$

The notations for the parameters are analogous to those used in Eq. 3.

In order to make a comparison with the formula derived by Omenetto et al., ${ }^{29}$ we established conditions identical to theirs; that is, the first-step transition in the two-step LEI mechanism is assumed to be optically saturated, while the optical excitation in the one-step LEI is undersaturated. Therefore, the ratio of $N_{i}$ (TLEI) to $N_{i}$ (SLEI) gives

$$
\frac{N_{i}(\text { TLEI })}{N_{i}(\text { SLEI })}=\alpha \frac{\lambda_{23}{ }^{2} A_{32} I_{23} k_{3 i} \Delta \omega_{12}}{\lambda_{12}{ }^{2} A_{21} I_{12} k_{2 i} \Delta \omega_{23}} .
$$

Equation 5 is found to be consistent with equation 40 obtained by Omenetto et al. Here $\alpha$ is relevant to the statistical weights, and $\Delta \omega_{23}$ and $\Delta \omega_{12}$ are equivalent to the depletion rate coefficients $\left(A_{32}+k_{32}+k_{3 i}\right)$ and $\left(A_{21}\right.$ $\left.+k_{21}+k_{2 i}\right)$, respectively, shown in their equation $40 .{ }^{29}$ Note that the laser linewidth is assumed to be very narrow, so that the convoluted transition linewidth observed is responsible for the effective lifetime of the states involved. The lifetime of the state is reciprocally proportional to the depletion rate of the state.

Since the conditions of first-step excitations in both one-step and two-step LEI schemes are considered to be identical, either in optical saturation or in linear interaction with radiation, the ratio of $N_{i}$ (TLEI) to $N_{i}$ (SLEI) follows:

$$
\begin{aligned}
R & =\frac{N_{i} \text { (TLEI) }}{N_{i} \text { (SLEI) }} \\
& =\left[\frac{\lambda_{23}{ }^{2} A_{32} I_{23} k_{3 i}}{2 \Pi \Delta \omega_{23}} N_{2} \Delta t\right] /\left[\frac{\lambda_{12}{ }^{2} A_{21} I_{12} k_{2 i}}{2 \Pi \Delta \omega_{12}} N_{1} \Delta t\right] \\
& =\frac{\lambda_{23}{ }^{2} A_{32} I_{23} k_{3 i}}{2 \Pi \Delta \omega_{23} k_{2 i}}
\end{aligned}
$$

where

$$
N_{2}=\frac{\lambda_{12}^{2} A_{21} I_{12}}{2 \Pi \Delta \omega_{12}} N_{1}
$$

analogously to Eq. 1 under the steady-state approximation. According to Eq. 6, the ion enhancement of TLEI over SLEI may be attributed to several factors such as second-step transition probability, laser intensity, collisional ionization rate coefficients, and transition linewidth, which is associated with the effective lifetime of the states involved in the transition. Note that Eq. 6 is equivalent to equation 36 given by Axner et al., ${ }^{31}$ ignoring the possibility of atom depletion during the laser pulse. According to the model derived by Axner et al., under the condition of neglect of the atom depletion effect, the ion enhancement between two-step LEI and one-step LEI becomes very weakly dependent upon the power of firststep laser excitation, when the power of second-step laser excitation is adjusted to be weak. Although the factor of atom depletion often comes to influence the ion enhancement induced by two-step LEI, it can be rendered negligible by an appropriate selection of the upper state for the second-step excitation, so that the laser pulse duration is shorter than the inverse of the collisional ionization rate from this state. ${ }^{31,32}$ In our model, for simplicity we assume that the laser pulse duration is very narrow, so that the convolution of the laser profile and the atomic profile can be considered as the atomic profile itself, for which the transition linewidth is measured by ignoring the contribution of the laser linewidth. In addition, the inverse of the collisional ionization rate $k_{3 i}$ becomes much larger than the laser pulse duration; therefore the requirement allowing neglect of the atom depletion can be fulfilled.

According to the formula derived by Lawton and Weinburg, ${ }^{39}$ the rate coefficient for collisional ionization $k_{i l}$ from a given analyte energy state $l$ can be expressed as

$$
k_{i l}=\sigma_{x l} \bar{v} n_{x} \exp [-\Delta E / k T]
$$

where $\sigma_{x l}$ is the partner-averaged cross section for collisional ionization from the state $l ; \bar{v}$, the relative average velocity of the analyte and the colliders in the flame; $n_{x}$, the number density of the collision partner; $T$, the flame temperature; and $\Delta E$, the energy difference between the ionization continuum and the state $l$. According to Eq. 8 , we may evaluate the collisional ionization rate coefficients involved in our system with $\mathrm{Ca}, \mathrm{Ba}$, and $\mathrm{K}$ as reagents. The collisional ionization rate coefficient $k_{3 i}$ for the case of Ca with ${ }^{3} P_{2}$ as the upper state may be estimated to be $2 \times 10^{7} \mathrm{~s}^{-1}$. The following data are used: The energy difference between the upper state and the ionization continuum is $10,752 \mathrm{~cm}^{-1}$, the collisional cross section of the $\mathrm{Ca}$ atom with the flame gases is assumed to be 100 $\AA^{2}$, and the flame temperature around $2500 \mathrm{~K}$ corresponds to the energy at $1740 \mathrm{~cm}^{-1}$. Note that the collisional cross section for ionization is hard to evaluate. We presume a value of $100 \AA^{2}$, approximately the same order of magnitude as the cross section of alkali atom with the flame gases adopted elsewhere, ${ }^{40,41}$ whereas this value is about ten times as large as the quenching cross section usually adopted for the low-lying excited analytes. For the case of $\mathrm{Ba}$ with ${ }^{3} P_{1}$ as the upper state, $k_{3 i}$ is estimated to be $1 \times 10^{8} \mathrm{~s}^{-1}$. Again, the collisional cross section of $\mathrm{Ba}^{*}$ with the flame gases is assumed to be $100 \AA^{2}$, and the energy difference between the upper state and the ionization continuum is $7209 \mathrm{~cm}^{-1}$. For the high-lying excited $\mathrm{K}$, the resulting $k_{3 i}$ is evaluated to be $4 \times 10^{9}$ $\mathrm{cm}^{-1}$ since the collisional cross section of $100 \AA^{2}$ and the energy difference of $1796 \mathrm{~cm}^{-1}$ are taken into account. Except for the high-lying $\mathrm{K}$ state, the inverse of the collisional ionization rate for $\mathrm{Ca}$ and $\mathrm{Ba}$ is larger than the laser pulse duration of 5 to $8 \mathrm{~ns}$ that is used. Therefore, the effect of atom depletion during the laser pulse seems to be insignificant in these two cases. 


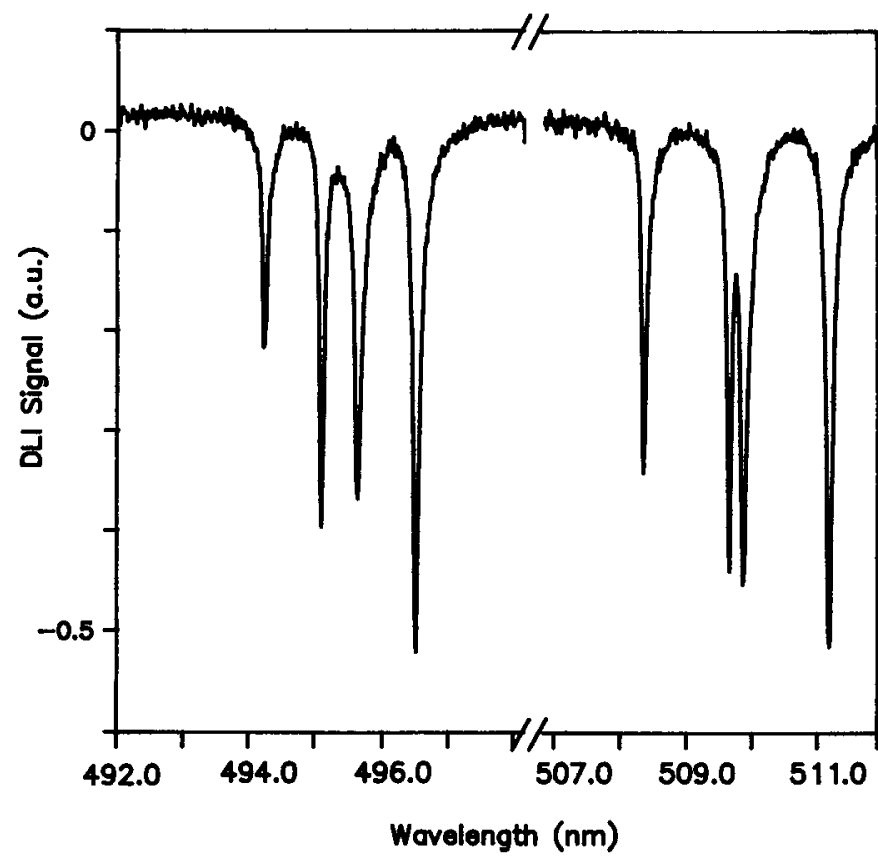

Fig. 2. Two-step LEI spectra for $100 \mathrm{ppm} \mathrm{K}$ atoms in an acetylene/ air flame. $\mathrm{K}$ atoms are first excited by the $4^{2} P_{1 / 2}$ (or $\left.4^{2} P_{3 / 2}\right)$ state, and then excited to either the $n^{2} S_{1 / 2}(n=9,10)$ or the $n^{2} D_{J}(n=7,8)$ state.

\section{RESULTS AND DISCUSSION}

Einstein Spontaneous Emission Coefficient. In order to examine the $A_{32}$ dependence of ion yield enhanced by the two-step LEI over the one-step LEI process, we have selected the $\mathrm{K}$ atom as the reagent, in which the $4^{2} S_{1 / 2} \rightarrow$ $4^{2} P_{1 / 2}\left(\right.$ or $\left.4^{2} P_{3 / 2}\right)$ transition is first excited, and then either the $4^{2} P_{1 / 2}\left(\right.$ or $\left.4^{2} P_{3 / 2}\right) \rightarrow n^{2} S_{1 / 2}$ or the $4^{2} P_{1 / 2}\left(\right.$ or $\left.4^{2} P_{3 / 2}\right) \rightarrow$ $n^{2} D_{J}$ transitions follow in the wavelength range from 492 to $512 \mathrm{~nm}$. The two-step LEI spectrum is shown in Fig. 2 , and the observed ion enhancement of TLEI/SLEI is presented in Table I. Because the two-step and one-step LEI schemes share a common intermediate state, $4^{2} P_{1 / 2}$ (or $4^{2} P_{3 / 2}$ ), and their conditions for first-step transition are identical, Eq. 6 may be employed to compare the potassium ion yields produced between the two-step and one-step LEI process.

While the first-step transition wavelength is fixed, ac- cording to Eq. 6, one obtains the ratio of ion enhancement of TLEI/SLEI as a function of second-step excitation as follows:

$$
\frac{R^{\prime}}{R}=\frac{k_{3 i}{ }^{\prime} \lambda_{23}{ }^{\prime \prime} A_{32}{ }^{\prime} I_{23}{ }^{\prime} / \Delta \omega_{23}{ }^{\prime}}{k_{3 i} \lambda_{23}{ }^{2} A_{32} I_{23} / \Delta \omega_{23}}
$$

In our experiment, the linewidth of each ionization profile resulting from the upper states, $n^{2} S_{1 / 2}$ or $n^{2} D_{J}$, as shown in Fig. 2, is roughly the same, the related wavelengths of second-step excitation are within a small range, and the laser intensity is normalized to be equal. Therefore Eq. 9 may be simplified to

$$
\frac{R^{\prime}}{R} \sim \frac{A_{32} k_{3 i}^{\prime}}{A_{32} k_{3 i}}
$$

It should be noted that these high-lying excited states are energetically close to each other, and thus the rates of collisional deactivation to the "neighborhood" states become so rapid $^{42,43}$ that the collisional ionization rates may be attributed to the summation of the ionization rates related to the neighborhood states. ${ }^{31}$ In addition, the energy difference between the high-lying excited states and the ionization continuum is very small, so that the total ionization rates may be partially attributed to the auto-ionization process induced by the electric field. ${ }^{38}$ Therefore, we consider the ionization rates stemming from these high-lying states to be roughly the same; i.e., $k_{3 i}{ }^{\prime} \sim$ $k_{3 i}$. The relative ion enhancement related to various stepwise excitation is thus determined by the ratio of the corresponding second-step transition probability.

In order to make a comparison with the experimental findings, we normalize the calculated ratio of TLEI/SLEI for the two-step LEI case with the second-step $4^{2} P_{1 / 2} \rightarrow$ $10^{2} S_{1 / 2}$ transition to its corresponding experimental value of $50 \pm 5$. According to Eq. 10 and the assumption of $k_{3 i}{ }^{\prime} \sim k_{3 i}$, estimation of the ion enhancement related to various second-step transitions, $4^{2} P_{1 / 2} \rightarrow n^{2} S_{1 / 2}(n=9$, $10)$ or $n^{2} D_{J}(n=7,8)$, is found to be in satisfactory agreement with our observation (Table I).

It is worthwhile to note that the ratio of TLEI/SLEI through different first-step transitions can also be predicted satisfactorily, except for the cases involving the $4^{2} P_{3 / 2} \rightarrow 9^{2} S_{1 / 2}$ or $4^{2} P_{3 / 2} \rightarrow 7^{2} D$, transitions as the secondstep excitation. For instance, the ratio related to the $4^{2} P_{3 / 2}$

TABLE I. Potassium ion enhancement of DLI/LEI. The calculated value is based on Eqs. 10 and 11 in the text, and then normalized to the

\begin{tabular}{|c|c|c|c|c|c|c|c|}
\hline \multirow{2}{*}{$\begin{array}{c}\text { Stepwise } \\
\text { transition }\end{array}$} & \multirow{2}{*}{$\begin{array}{l}\text { State } \\
\text { energy } \\
\left(\mathrm{cm}^{-1}\right)\end{array}$} & \multirow{2}{*}{$\begin{array}{l}\text { Second-step } \\
\text { wavelength } \\
(\mathrm{nm})\end{array}$} & \multirow{2}{*}{$\begin{array}{c}A_{32^{\mathrm{a}}} \\
\left(10^{8} \mathrm{~s}^{-1}\right)\end{array}$} & \multirow[b]{2}{*}{$n^{* \mathrm{~b}}$} & \multirow[b]{2}{*}{$\mu^{\mathrm{c}}$} & \multicolumn{2}{|c|}{ Ion enhancement } \\
\hline & & & & & & $\operatorname{ExP}^{d}$ & Cal. \\
\hline $\begin{array}{l}4^{2} P_{1 / 2} \rightarrow 10^{2} S_{1 / 2} \\
4^{2} P_{3 / 2} \rightarrow 10^{2} S_{1 / 2} \\
4^{2} P_{1 / 2} \rightarrow 9^{2} S_{1 / 2} \\
4^{2} P_{3 / 2} \rightarrow 9^{2} S_{1 / 2}\end{array}$ & $\begin{array}{l}33214.4 \\
33214.4 \\
32648.2 \\
32648.2\end{array}$ & $\begin{array}{l}494.20 \\
495.61 \\
508.43 \\
509.92\end{array}$ & $\begin{array}{l}0.00213 \\
0.00425 \\
0.00350 \\
0.00700\end{array}$ & $\begin{array}{l}7.83 \\
7.83 \\
6.82 \\
6.82\end{array}$ & $\begin{array}{l}2.18 \\
2.18 \\
2.18 \\
2.18\end{array}$ & $\begin{array}{r}50 \\
95 \\
89 \\
115\end{array}$ & $\begin{array}{r}50 \\
100 \\
82 \\
164\end{array}$ \\
\hline $\begin{array}{l}4^{2} P_{1 / 2} \rightarrow 8^{2} D_{3 / 2} \\
4^{2} P_{3 / 2} \rightarrow 8^{2} D_{3 / 2.5 / 2} \\
4^{2} P_{1 / 2} \rightarrow 7^{2} D_{2 / 3} \\
4^{2} P_{3 / 2} \rightarrow 7^{2} D_{3 / 2.5 / 2}\end{array}$ & $\begin{array}{l}33178.4 \\
33178.4 \\
32598.5 \\
32598.5\end{array}$ & $\begin{array}{l}495.08 \\
496.50 \\
509.72 \\
511.22\end{array}$ & $\begin{array}{l}0.00220 \\
0.0026 \\
0.0029 \\
0.0035\end{array}$ & $\begin{array}{l}7.74 \\
7.74 \\
6.75 \\
6.75\end{array}$ & $\begin{array}{l}0.26 \\
0.26 \\
0.25 \\
0.25\end{array}$ & $\begin{array}{r}94 \\
145 \\
106 \\
145\end{array}$ & $\begin{array}{l}100 \\
177 \\
132 \\
238\end{array}$ \\
\hline
\end{tabular}
experimental result for the transition at $4^{2} P_{1 / 2} \rightarrow 10^{2} S_{1 / 2}$.

${ }^{a}$ Einstein spontaneous emission coefficient; See ref. 44.

${ }^{b}$ Defined as $n-\mu ; n$ is the principal quantum number and $\mu$ is the quantum defect. Its value can be calculated by $1 / \sqrt{\epsilon} ; \epsilon$, in units of Rydberg, indicates the energy required to reach the ionization continuum.

- Quantum defect.

Relative error $=10 \%$. 


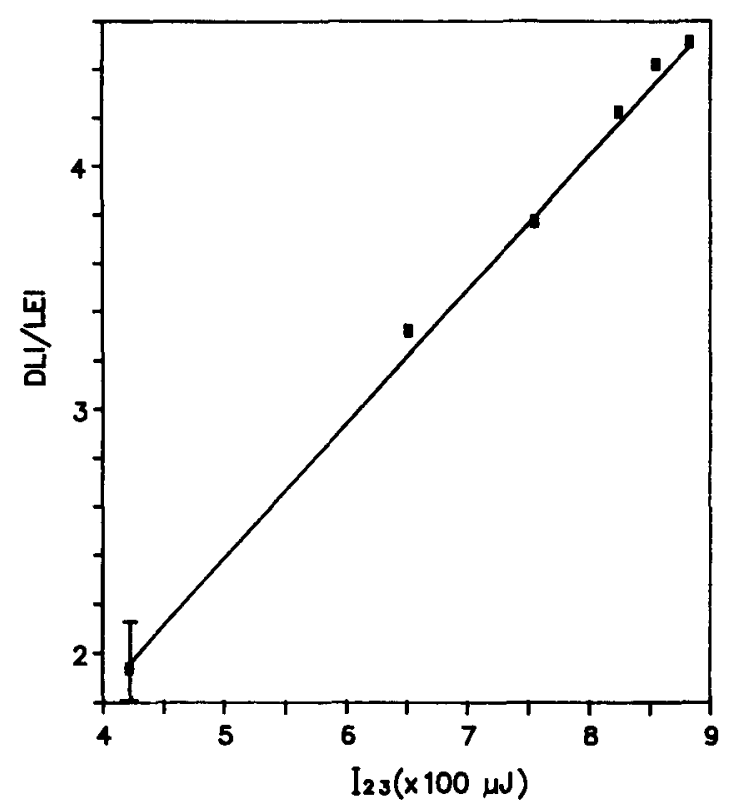

FIG. 3. Measurement of $\mathrm{Ca}$ ion enhancement of TLEI/SLEI as a function of the second-laser intensity, used to excite the $4 s 4 p^{3} P_{1} \rightarrow 4 p^{23} P_{2}$ transition.

$\rightarrow 10^{2} S_{1 / 2}$ transition is estimated to be 100 , consistent with the observed $95 \pm 10$ value. To deal with this case, one should multiply a factor of the state-degeneracy ratio $g_{3} / g_{2}$ by the right-hand side of Eq. $6 .^{38}$ Assuming that $k_{3 i}{ }^{\prime}$ $\sim k_{3 i}$, Eq. 10 becomes

$$
\frac{R^{\prime}}{R} \sim \frac{g_{3}{ }^{\prime} g_{2} A_{32}{ }^{\prime}}{g_{2}{ }^{\prime} g_{3} A_{32}} .
$$

Taking into account the degeneracy of states involved in the transitions and the respective first-step transition probability, we estimate the ratio of TLEI/SLEI via the $4^{2} P_{3 / 2} \rightarrow 10^{2} S_{1 / 2}$ transition to be twice as large as that obtained via the $4^{2} P_{1 / 2} \rightarrow 10^{2} S_{1 / 2}$ transition. Analogously, the ratios of TLEI/SLEI via the $4^{2} P_{1 / 2} \rightarrow 8^{2} D_{3 / 2}$ and $4^{2} P_{3 / 2}$ $\rightarrow 8^{2} D_{J}$ transitions are estimated to be 100 and 177 , in agreement with the observed values $94 \pm 10$ and $145 \pm$ 15, although the related $A_{32}$ values appear to be almost equal. The same values can alternatively be evaluated by Eq. 5, which must include the state degeneracy as correction.

The probable causes for the discrepancy in terms of the model prediction may be complicated. As discussed above, the model is not sufficiently perfect to account for the excited $\mathrm{K}$ atom depletion during the laser pulse duration. As a result, the ion enhancement of TLEI/SLEI may become dependent upon the power of the first-step excitation laser, which is neglected in our model. ${ }^{31,32}$ In addition, the Einstein spontaneous emission coefficient $A_{32}$, as reported in Ref. 44, bears an uncertainty of 25 to $50 \%{ }^{44}$ These considerations may partially explain the deviation observed in a comparison of ion enhancement results.

It is obvious that the Einstein spontaneous emission coefficient will decrease with an increase in the principal quantum number, $n$. The transition probability is reported to be approximately proportional to $1 / n^{* 3} \cdot{ }^{45} n^{*}$ is the effective principal quantum number, equal to $n-\mu$
( $\mu$ is the quantum defect). ${ }^{34,45}$ The $A_{32}$ values listed in Table I agree with this prediction. Axner et al. have demonstrated that the ionization signals of two-step LEI for $\mathrm{Na}, \mathrm{Li}$, and $\mathrm{Tl}$ keep decreasing with an increase in the principal quantum number around the ionization continuum. ${ }^{46}$ Therefore, the likely intuitive concept - that the state which is excited stepwise closer to the ionization continuum causes a larger ion enhancement-is not true.

Laser Intensity. The Ca atom has been selected for this test. The two-step LEI scheme of Ca shown in Fig. $1 \mathrm{~b}$ is composed of a stepwise excitation via the $4 s^{21} S_{0} \rightarrow 4 s 4 p^{3} P_{1}$ and $4 s 4 p^{3} P_{1} \rightarrow 4 p^{23} P_{2}$ transitions, while the one-step LEI scheme reflects only the $4 s^{21} S_{0} \rightarrow 4 s 4 p^{3} P_{1}$ transition. As the laser intensity in the first-step excitation is fixed, the ratio of two-step to one-step LEI signal is measured as a function of laser intensity of the second-step excitation, and thereby shows a straight line of slope equal to unity (Fig. 3). The result suggests that the second laser intensity in the two-step LEI scheme plays an important role in the optimization of the technique. According to Eq. 6, since the conditions of the $\lambda_{23}, A_{32}, k_{3 i}, \Delta \omega_{23}$, and $k_{2 i}$ parameters remain invariant, the ratio $R$ becomes proportional to $I_{23}$. Our observation is consistent with the theoretical prediction. The result in Fig. 3 also indicates that the collisional ionization mechanism is dominant over the photoionization process. Through the latter process, the atoms populated in the upper state are ionized by absorbing an additional photon from either the first laser or the second laser. Because the photoionization cross section decays almost exponentially with an increase of overshoot of the laser above the ionization continuum, a high-lying state excited closer to the ionization continuum bears a smaller photoionization cross section. ${ }^{47}$ Using $\mathrm{Na}$ and $\mathrm{Li}$ as examples, Axner and Sjostrom have made a comparison between the two-step LEI and photoionization techniques and have found that the former technique is several orders of magnitude more sensitive than the latter. ${ }^{48}$

Collisional Ionization Rate Coefficient. We also have selected the $\mathrm{Ca}$ atom as the reagent for examing the influence of the $k_{2 i}$ factor on the ion enhancement by twostep LEI. As shown in Fig. 1b, two ionization schemes of the two-step LEI process for the Ca atom have been used; one process involves the $4 s^{21} S_{0} \rightarrow 4 s 4 p^{3} P_{1}$ transition at $657.3 \mathrm{~nm}$ as the first-step excitation and the $4 s 4 p^{3} P_{1}$ $\rightarrow 4 p^{23} P_{2}$ transition at $428.3 \mathrm{~nm}$ as the second-step excitation, and the other process is via the $4 s^{21} S_{0} \rightarrow 4 s 4 p^{1} P_{1}$ transition at $422.7 \mathrm{~nm}$ and then the $4 s 4 p^{1} P_{1} \rightarrow 4 p^{23} P_{2}$ transition at $671.5 \mathrm{~nm}$. Both schemes share a common upper $4 p^{23} P_{2}$ state. According to Eq. 6 , the relative ion enhancement $R^{\prime} / R$ for these two schemes is expressed as

$$
\frac{R^{\prime}}{R}=\frac{\lambda_{23}{ }^{\prime \prime} A_{32}{ }^{\prime} I_{23}{ }^{\prime} k_{3 i}{ }^{\prime}}{2 \Pi \Delta \omega_{23}{ }^{\prime} k_{2 i}{ }^{\prime}} / \frac{\lambda_{23}{ }^{2} A_{32} I_{23} k_{3 i}}{2 \Pi \Delta \omega_{23} k_{2 i}} .
$$

$R^{\prime}$ and $R$ indicate the ratio of TLEI/SLEI for the ${ }^{1} S_{0}-{ }^{3} P_{1}-$ ${ }^{3} P_{2}$ and ${ }^{1} S_{0}-1 P_{1}-{ }^{3} P_{2}$ process, respectively. Because the intermediate states ${ }^{3} P_{1}$ or ${ }^{1} P_{1}$ have the same degeneracy, we omit the degeneracy correction in Eq. $12 . k_{3 i}{ }^{\prime}=k_{3 i}$ since the upper states are identical; $\Delta \omega_{23}{ }^{\prime} \sim \Delta \omega_{23}$ from the experimental comparison of the transition linewidth of ionization profiles; and $k_{2 i} / k_{2 i}{ }^{\prime}=\exp \left[-\left(E_{2}-E_{2}{ }^{\prime}\right) / k T\right]$, assuming that the partner-averaged cross sections for 
TABLE II. Experimental measurement and theoretical calculation of relative ion enhancement $R^{\prime} / R$ as a function of collisional ionization rate coefficient and transition linewidth. The parameters $\lambda_{1}$ and $\lambda_{2}$ indicate the first-step and the second-step transitions; $A_{32}$ is the second-step Einstein spontaneous emission coefficient, and $I_{23}$ is the second-step laser intensity. The calculated values of $R^{\prime} / R$ are based on Eqs. 13 and 14 in the text.

\begin{tabular}{|c|c|c|c|c|c|c|c|c|}
\hline $\begin{array}{l}\text { Ele- } \\
\text { ment }\end{array}$ & $\begin{array}{l}\text { First-step } \\
\text { transition }\end{array}$ & $\begin{array}{l}\text { Second-step } \\
\text { transition }\end{array}$ & $\begin{array}{c}\lambda_{1} \\
(\mathrm{~nm})\end{array}$ & $\begin{array}{c}\lambda_{2} \\
(\mathrm{~nm})\end{array}$ & $\begin{array}{c}A_{32^{\mathrm{a}}} \\
\left(10^{8} \mathrm{~s}^{-1}\right)\end{array}$ & $\begin{array}{c}I_{23} \\
(\mu \mathrm{J})\end{array}$ & $\left(R^{\prime} / R\right)_{\exp }$ & $\left(R^{\prime} / R\right)_{\mathrm{cal}}$ \\
\hline $\mathrm{Ca}$ & $4 s^{21} S_{0} \rightarrow 4 s 4 p^{3} P_{1}$ & $4 s 4 p^{3} P_{1} \rightarrow 4 p^{23} P_{2}$ & 657.28 & 428.30 & 0.434 & $680 \pm 40$ & $72 \pm 12$ & 74 \\
\hline \multirow[t]{2}{*}{$\mathrm{Ba}$} & $6 s^{21} S_{0} \rightarrow 6 s 6 p^{3} P_{1}$ & $6 s 6 p^{3} P_{1} \rightarrow 6 p^{23} P_{1}$ & 791.14 & 450.59 & 1.1 & $430 \pm 30$ & \multirow{2}{*}{$0.98 \pm 0.28$} & \multirow{2}{*}{0.4} \\
\hline & $6 s^{21} S_{0} \rightarrow 6 s 6 p^{3} P_{1}$ & $6 s 5 d^{1} D_{2} \rightarrow 6 s 4 f^{1} F_{3}$ & 791.14 & 428.31 & 0.64 & $234 \pm 20$ & & \\
\hline
\end{tabular}

${ }^{a}$ See Ref. 44 ; uncertainty within 25 to $50 \%$.

ionization either from the $N_{2}{ }^{\prime}\left({ }^{3} P_{1}\right)$ or the $N_{2}\left({ }^{1} P_{1}\right)$ state are identical. Therefore, Eq. 10 is written as

$$
\frac{R^{\prime}}{R}=\frac{\lambda_{23}{ }^{2 \prime} A_{32}{ }^{\prime} I_{23}{ }^{\prime}}{\lambda_{23}{ }^{2} A_{23} I_{23}} \exp \left[-\left(E_{2}-E_{2}{ }^{\prime}\right) / k T\right] \text {. }
$$

Given, in Table II, the literature values of $\lambda_{23}, A_{32}, \lambda_{23}{ }^{\prime}$, and $A_{32}{ }^{\prime}$ as well as $I_{23}$ and $I_{23}{ }^{\prime}$ measured in our experiment, we estimate an $R^{\prime} / R$ ratio of 74 , in agreement with our observation of relative ion enhancement, $72 \pm 12$. This fact indicates that the ion yield ratio of TLEI and SLEI may be enhanced when a small value of $k_{2 i}$ is selected. The two-step LEI technique may be performed more effectively for those species with a larger ionization continuum, from which a larger energy difference in the state excited leads to a smaller collisional ionization rate; thus the sensitivity of the one-step LEI scheme may be reduced. In contrast, for increasing the detection sensitivity of LEI with single-laser excitation, it is advisable to select an excited state close to the ionization continuum, provided that the transition probability keeps reasonably large. This is the strategy adopted by Axner et al. to successfully demonstrate the multielement-detection capability of one-step LEI using UV light as an excitation source for reducing the energy difference from the ionization continuum. ${ }^{49}$

Linewidth of the Transition. According to the uncertainty principle, the linewidth of the transition may correspond to the reciprocal of the effective lifetime of the states involved in the transition, assuming that the laser linewidth is very narrow and negligible. A decrease in the transition linewidth causes an increase in the effective lifetime for the state. Thus this factor is also equivalent to the total depletion rate coefficient reported by Omenetto et al., ${ }^{29}$ as discussed above. Because measuring the depletion rate coefficient accurately in a flame system is not easy, it appears to be practical to use the transition linewidth to represent this rate coefficient to determine its role in the two-step LEI optimization.

Considering the specific nature of its intermediate state, the $\mathrm{Ba}$ atom is selected as the reagent for the $\Delta \omega_{23}$ test. In the first two-step LEI scheme employed (Fig. 1c), the $\mathrm{Ba}$ atom was excited from the $6 s^{21} S_{0}$ to the $6 s 6 p^{3} P_{1}$ state at $791.1 \mathrm{~nm}$, followed by absorption of a second photon to $6 p^{23} P_{1}$ at $450.6 \mathrm{~nm}$. These two laser beams were overlapped spatially and temporally above the center of the burner head. In contrast, in the second two-step LEI scheme, the second laser was optically delayed by $2 \mathrm{~ns}$ with respect to the first laser and used to excite the $6 s 5 d^{1} D_{2}$ $\rightarrow 6 s 4 f^{1} F_{3}$ transition at $428.3 \mathrm{~nm}$, while the transition of the first-step excitation was kept invariant. Comparing the relative ion enhancement of the first TLEI scheme with the second TLEI scheme, one obtains the following equation according to Eq. 6 with correction of state degeneracy taken into account:

$$
\begin{aligned}
\frac{R^{\prime}}{R} & =\left(\frac{g_{3}{ }^{\prime}}{g_{2}{ }^{\prime}}\right) \frac{\lambda_{23}{ }^{2 \prime} A_{32}{ }^{\prime} I_{23}{ }^{\prime} k_{3 i}{ }^{\prime}}{2 \Pi \Delta \omega_{23}{ }^{\prime} k_{2 i}{ }^{\prime}} /\left(\frac{g_{3}}{g_{2}}\right) \frac{\lambda_{23}{ }^{2} A_{32} I_{23} k_{3 i}}{2 \Pi \Delta \omega_{23} k_{2 i}} \\
& =\left(\frac{g_{3}{ }^{\prime} g_{2}}{g_{2}{ }^{\prime} g_{3}}\right) \frac{\lambda_{23}{ }^{2} A_{32}{ }^{\prime} I_{23}{ }^{\prime} k_{3 i}{ }^{\prime}}{\lambda_{23}{ }^{2} A_{32} I_{23} k_{3 i}}\left(\frac{\Delta \omega_{23}}{\Delta \omega_{23}{ }^{\prime}}\right) .
\end{aligned}
$$

Here $R^{\prime}$ and $R$ indicate the ratio of TLEI/SLEI induced by the first and the second two-step LEI schemes, respectively. In the above equation, $k_{2 i}=k_{2 i}{ }^{\prime}$, because the one-step LEI processes involved in both cases are identical. For making a quantitative comparison with the experimental results, we adopt the following assumptions: (1) $k_{3 i}{ }^{\prime} \sim k_{3 i}$, because the upper states $6 p^{23} \mathrm{P}_{1}$ and $6 s^{4} 4 f^{1} F_{3}$ differ by less than $100 \mathrm{~cm}^{-1}$. (2) The population densities of the intermediate states $6 s 6 p^{3} P_{1}$ and $6 s 5 d^{1} D_{2}$ are assumed to be identical. Given the parameters in Table II, in which $\Delta \omega_{23} / \Delta \omega_{23}{ }^{\prime}$ was measured to be $0.16 \pm 0.03$ and $I_{23}$ and $I_{23}{ }^{\prime}$ were $234 \pm 20 \mu \mathrm{J}$ and $430 \pm 30 \mu \mathrm{J}$, respectively, we evaluate $R^{\prime} / R$ to be 0.4 . Our observation of $R^{\prime} / R$ was $0.98 \pm 0.28$. Although the comparison seems to be in disagreement, the predicted value is expected to be smaller than that observed. Because there are several channels available for depleting the population of the $6 s 6 p^{3} P_{1}$ intermediate state, the population distributed to the $6 s 5 d^{1} D_{2}$ state should be less than that promoted initially. The $R$ value of the second two-step LEI scheme is very sensitive to the delay time between these two lasers. As the delay time was adjusted from 0 to $10 \mathrm{~ns}$, the TLEI signal appeared to be at a maximum at the 2-ns delay. Without knowledge of the branching ratio of the population separating into the $6 s 5 d^{1} D_{2}$ state, it is hard to estimate how much population is still left in this state at a 2-ns delay, and therefore a precise quantitative comparison becomes impossible.

In summary, two-step LEI is, in general, believed to be more sensitive and selective in trace analysis than is one-step LEI. As reported previously, for optimization of the two-step LEI configuration, the factors involving the second laser intensity, the transition probability of second-step excitation, the collisional ionization rates, and the transition linewidth (or quenching efficiency toward the excited states) should be taken into account. In this work, we have derived a simple theoretical model in relation to these parameters, that is rough but is suitable in practice for characterizing the ion yield enhanced by 
two-step over one-step LEI. According to this model, each parameter affecting the ion enhancement of TLEI/SLEI has been systematically examined and compared to its theoretical evaluation. The findings are consistent with the model prediction, except for the effect of transition linewidth, which is underestimated theoretically due to the difficulty of establishing experimental conditions.

\section{ACKNOWLEDGMENT}

This work is financially supported by the National Science Council of the Republic of China under Contract No. NSC 82-0208-M001-087.

1. J. C. Travis, G. C. Turk, and R. B. Green, Anal. Chem. 54, 1006A (1982).

2. G. C. Turk and N. Omenetto, Appl. Spectrosc. 40, 1085 (1986).

3. B. S. Smith, L. Hart, and N. Omenetto, Anal. Chem. 58, 2147 (1986).

4. K. D. Su, K. C. Lin, W. T. Luh, Appl. Spectrosc. 46, 1370 (1992).

5. E. G. Mallard and K. C. Smyth, Comb. Flame 44, 61 (1982).

6. K. C. Lin, P. M. Hunt, and S. R. Crouch, Chem. Phys. Lett. 90, $111(1982)$.

7. K. D. Su, C. Y. Chen, K. C. Lin, and W. T. Luh, Appl. Spectrosc. 45, 1340 (1991).

8. W. G. Mallard, J. Houston, and K. C. Smyth, J. Chem. Phys. 76, 3483 (1982).

9. O. Axner and T. Berglind, Appl. Spectrosc. 40, 1224 (1986).

10. T. A. Cool and P. J. H. Tjossem, in Gas-Phase Chemiluminescence and Chemi-ionization, A. Fontijn, Ed. (Elsevier Science Publishers, Amsterdam, 1985), pp. 105-116.

11. T. A. Cool and J. E. M. Goldsmith, Appl. Opt. 26, 3542 (1987).

12. K. S. Epler, T. C. O'Haver, G. C. Turk, and W. A. MacCrehan, Anal. Chem. 60, 2062 (1988)

13. T. Berglind, S. Nilsson, and H. Rubinsztein-Dunlop, Phys. Scripta 36, 246 (1987).

14. R. B. Green, G. J. Havrilla, and T. O. Trask, Appl. Spectrosc. 34, $561(1980)$.

15. G. J. Havrilla and C. C. Carter, Appl. Opt. 26, 3510 (1987).

16. O. Axner, T. Berglind, J. I. Heully, I. Lingren, and H. RubinszteinDunlop, J. Appl. Phys. 55, 3215 (1984).

17. T. A. Cool, Appl. Opt. 23, 1559 (1984)

18. G. C. Turk, Anal. Chem. 53, 1187 (1981).

19. G. J. Havrilla and R. B. Green, Anal. Chem. 52, 2376 (1980).

20. M. A. Nippoldt and R. B. Green, Anal. Chem. 55, 554 (1983).

21. G. J. Havrilla, P. K. Schenck, J. C. Travis, and G. C. Turk, Anal. Chem. 56, 186 (1984).

22. G. C. Turk, Anal. Chem. 64, 1836 (1992).
23. O. Axner, M. Lejon, I. Magnusson, H. Rubinsztein-Dunlop, and S. Sjostrom, Appl. Opt. 26, 3521 (1987).

24. N. Omenetto, T. Berthoud, P. Cavalli, and G. Rossi, Anal. Chem. 57, $1256(1985)$

25. I. Magnusson, O. Axner, and H. Rubinsztein-Dunlop, Phys. Scripta 33, 429 (1986).

26. G. C. Turk, Anal. Chem. 63, 1607 (1991).

27. K. C. Lin, S. H. Lin, P. M. Hunt, G. E. Leroi, and S. R. Crouch, Appl. Spectrosc. 43, 68 (1989).

28. G. J. Havrilla and K. J. Choi, Anal. Chem. 58, 3095 (1986).

29. N. Omenetto, B. W. Smith, and L. P. Hart, Fresenius Z. Anal. Chem. 324, 683 (1986).

30. O. Axner, T. Berglind, J. L. Heully, I. Lindgren, and H. RubinszteinDunlop, J. Appl. Phys. 55, 3215 (1984).

31. O. Axner, M. Norberg, and H. Rubinsztein-Dunlop, Spectrochim. Acta 44B, 693 (1989).

32. O. Axner and H. Rubinsztein-Dunlop, Spectrochim. Acta 44B, 837 (1989).

33. K. C. Lin and Y. S. Duh, Appl. Spectrosc. 43, 20 (1989).

34. O. Axner and S. Sjostrom, Appl. Spectrosc. 44, 864 (1990).

35. K. C. Lin, Y. S. Duh, T. C. Sheu, and T. J. Hsu, J. Chin. Chem. Soc. 35, 179 (1988).

36. L. Volk, W. Richardson, K. H. Lau, and S. H. Lin, J. Chem. Educ. 54, 95 (1977).

37. J. C. Travis, J. Chem. Educ. 59, 909 (1982)

38. V. S. Letokhov, Nonlinear Laser Chemistry (Springer-Verlag, Berlin, 1983), Chap. 3

39. J. Lawton and F. J. Weinburg, Electrical Aspects of Combustion (Clarendon Press, Oxford, 1969), 1st ed. p. 231.

40. K. C. Smyth, P. K. Schenck, and W. G. Mallard, in Laser Probes for Combustion Chemistry, D. R. Crosley, Ed. (American Chemical Society, Washington, D.C., 1980), pp. 175-181.

41. Tj. Hollander, AIAA Journal 6, 385 (1968).

42. Y. L. Luo, K. C. Lin, D. K. Liu, and W. T. Luh, Phys. Rev. A 46, 3834 (1992).

43. H. C. Chang, Y. L. Luo, and K. C. Lin, J. Chem. Phys. 94, 3529 (1991).

44. W. L. Wiese, M. W. Smith, and B. M. Miles, Atomic Transition Probabilities (National Bureau of Standards, Gaithersburg, Maryland, 1969), Vol. II.

45. C. Fabre and S. Haroche, in Rydberg States of Atoms and Molecules, R. F. Stebbings and F. B. Dunning, Eds. (Cambridge University Press, London, 1983), pp. 117-164.

46. O. Axner, T. Berglind, and S. Sjostrom, Phys. Scripta 34, 18 (1986).

47. F. M. Curran, K. C. Lin, G. E. Leroi, P. M. Hunt, and S. R. Crouch, Anal. Chem. 55, 238 (1983).

48. O. Axner and S. Sjostrom, Appl. Spectrosc. 44, 144 (1990).

49. O. Axner, I. Magnusson, J. Petersson, and S. Sjostrom, Appl. Spectrosc. 41, 19 (1987) 